\title{
Multi-beam energy acquisition in FE-EPMA
}

\author{
Silvia Richter and Philippe T. Pinard
}

Central Facility for Electron Microscopy, RWTH Aachen University, Aachen, Germany.

Varying the beam energy is a common method used for the analysis of laterally homogeneous multilayered structures in EPMA. With increasing beam energy the information depth is increasing as well. Thus, the multi beam energy acquisition delivers a possibility for depth profiling [1]. On the other side, cross sections measurements are often performed at one single beam energy. This guarantees stable conditions like well-adjusted focus, astigmatism and gun alignment. Furthermore, available quantification software is mainly designed for single beam energy acquisition and for the accurate determination of the lateral resolution the lateral ionisation probability is less studied and proven than the well-known $\varphi(\rho z)$-function.

With the introduction of the field emission electron microprobes the analysis of submicron features have been becoming more and more of interest due to better focusing capabilities. Since the spatial resolution is then mainly determined by the broadening of the beam due to scattering events within the specimen, a common solution is to apply low voltage analysis, i.e. the use of one single low beam energy. However, as shown recently [2] the low voltage analysis has its limitations, if soft X-ray lines, i.e. L $\alpha$ lines of the transmission elements, are used. Thus, the goal of our work is to present a new technique, where the acquisition is performed at multiple beam energies using well-established X-ray lines and almost the same overvoltage ratios.

For the proper selection of the beam energies the lateral intensity distribution was studied by means of Monte Carlo simulations in MONACO [3]. The features of interest are needle-like carbides in high manganese steel (Fig. 1) containing the binding elements Fe, Mn and Al. A minimum beam energy of 9 $\mathrm{keV}$ is needed to excite the highest energetic line: Fe K $\alpha$. However, at this beam energy the soft X-ray lines, $\mathrm{Al} \mathrm{K} \alpha$ and $\mathrm{C} \mathrm{K} \alpha$, limit the lateral resolution to about $600 \mathrm{~nm}$ (see Fig. 2). Lowering the beam energy down to $4 \mathrm{keV}$ and keeping in mind from previous work [4], that the beam diameter is worsen at lower beam energy, the lateral resolution of $\mathrm{Al} \mathrm{K} \alpha$ and $\mathrm{C} \mathrm{K} \alpha$ was adapted to the resolution of the other ones (see Fig. 3). At this well-chosen set of conditions the measurement was performed by scanning the beam across the precipitate. In between the two conditions the beam was focused and positioned manually. After background subtraction and calibration k-ratios were extracted from the maximum of the line profile and converted into elemental concentration using a special matrix correction procedure (see Table 1).

One step forward an automotive procedure was developed for a series of high spatial resolution measurements where each position, i.e. precipitate, on the sample is analyzed under different conditions. The challenges are related to the stage reproducibility, beam drift, focusing capabilities and positioning of the beam [4]. Especially, changing the beam energy results in a large shift of the beam of up to several $\mu \mathrm{m}$ (Fig. 4). A shift correction procedure is proposed based on image registration techniques. The basic principles are shown in Fig. 5, where a reference image is compared by a crosscorrelation function with images taken at 4 and $10 \mathrm{keV}$. The goal of the analyses was to quantify the chemical composition of small $\mathrm{Nb}$-enriched precipitates in a low alloyed steel matrix (see Table 2). Fe $\mathrm{K} \alpha$ and $\mathrm{Cr} \mathrm{K} \alpha$ were analysed at $10 \mathrm{keV}$ and the other $\mathrm{X}$-ray lines at $4 \mathrm{keV}$. Since contrast and brightness values of backscattered electron images are highly sensitive to condition changes, limits of the new measurement strategy are also presented. 
[1] P. Willich, Mikrochim. Acta 12 (1992), pp.1-17.

[2] P. Pinard et al, Micros. Microanal. 20 S3 (2014), pp. 700-701.

[3] N. Ammann, P. Karduck, Microbeam Analysis (1990) (J. R. Michael, P. Ingram, eds.), San Francisco Press, San Francisco, p. 150.

[4] S. Richter et al, Microsc. Microanal. 20 S2 (2014), pp. 680-681.
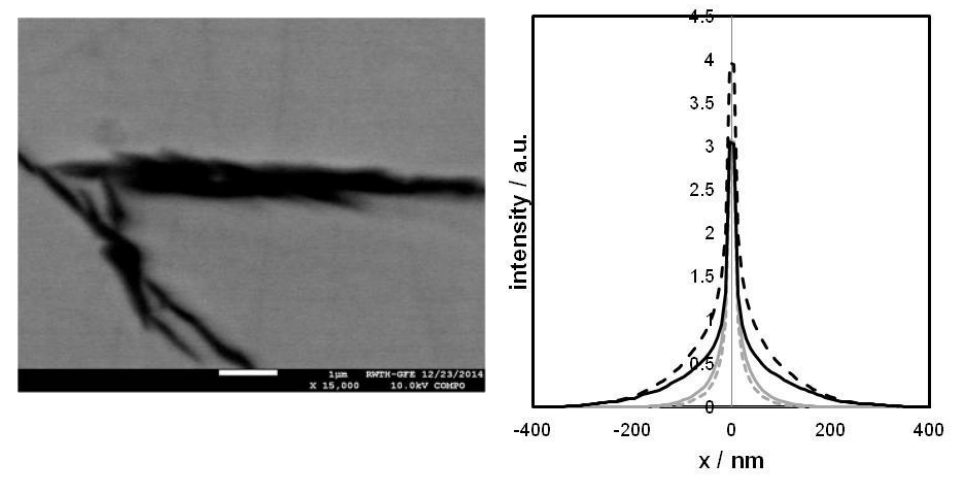

Figure 2: Lateral intensity

Figure 1: BSE-image of high Mn steel with carbides.

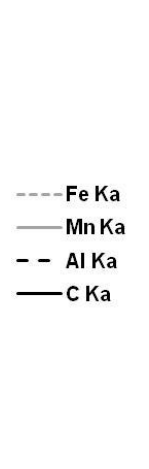

distributions at $9 \mathrm{keV}$

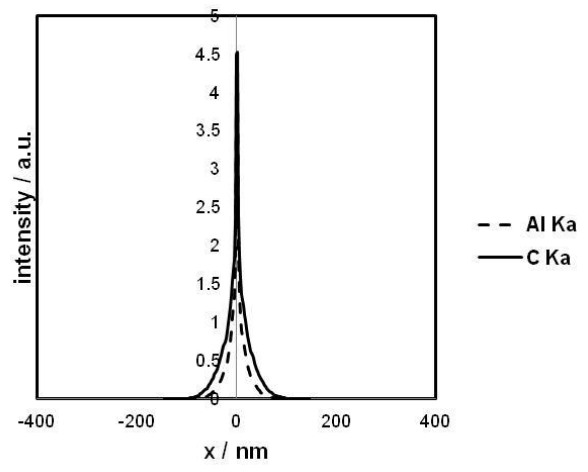

Figure 3: Lateral intensity distributions at $4 \mathrm{keV}$

Table 1: Composition of carbide shown in Fig. 1and root mean square deviation between measured and simulated k-ratios.

\begin{tabular}{|c|c|c|c|c|c|}
\hline & Fe & Mn & Al & C & RMS $\left(\mathbf{k}_{\text {exp }}, \mathbf{k}_{\text {th }}\right)$ \\
\hline at $\%$ & 55.8 & 18.2 & 12.5 & 13.5 & $1.03 \%$ \\
\hline
\end{tabular}

Figure 4 (left): Shift of the beam position as a

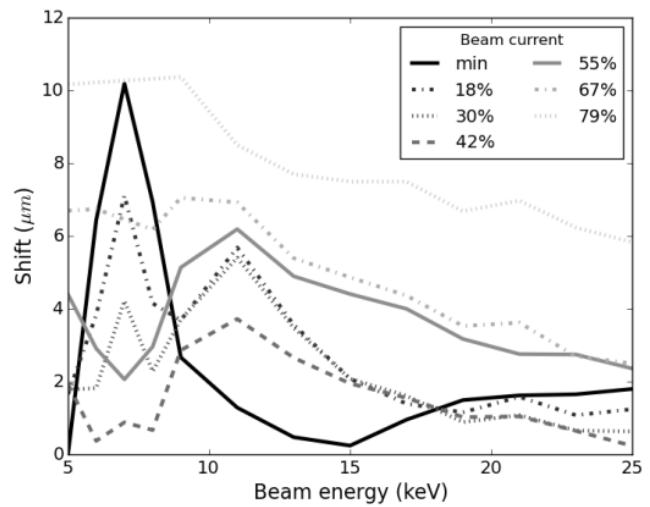
function of the beam energy at different beam currents

\begin{tabular}{|c|c|c|c|c|c|c|}
\hline & $\mathrm{Si}$ & $\mathrm{Cr}$ & $\mathrm{Fe}$ & $\mathrm{Nb}$ & Mo & Total \\
\hline wt\% & 3.7 & 5.3 & 50.9 & 30.9 & 8.6 & 99.2 \\
\hline
\end{tabular}

Table 2: Composition of Nb-enriched precipitate
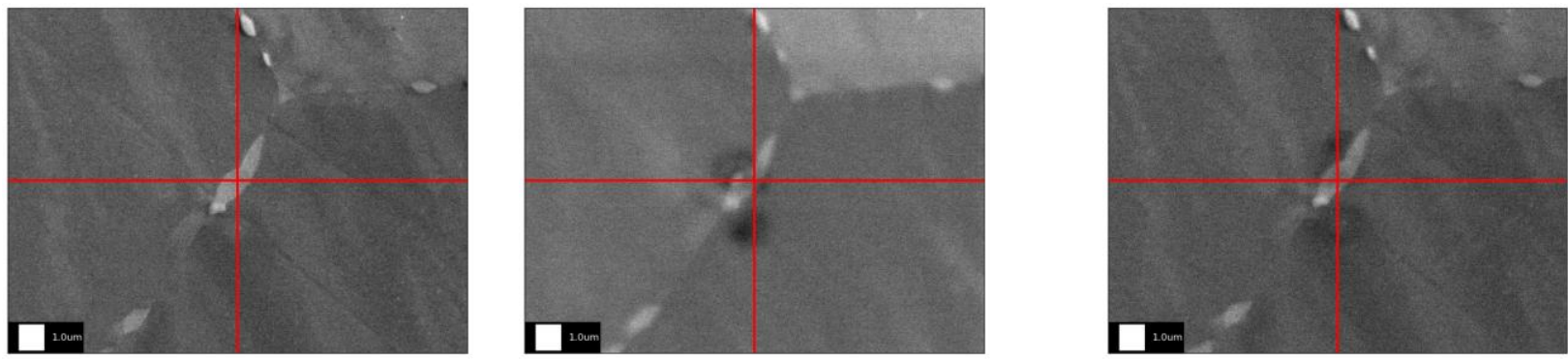

Figure 5: Principle of image registration technique using a reference image (left) for comparison with images acquired at $4 \mathrm{keV}$ (middle) and $10 \mathrm{keV}$ (right). 